\title{
Boundary Element Method for Calculating the Pressure of Heterogeneous Reservoir
}

\author{
Y. A. Zhang ${ }^{1, *}$, J. F. Jia ${ }^{2}$, H. W. Wang ${ }^{1, *}$, B. S. Li ${ }^{1}$, Y. R. Xu ${ }^{1}$ \\ ${ }^{1}$ Pctroleum Production Technology Research Institute, Jilin Oilfield Company, Songyuan, Jilin 131200 , \\ China \\ ${ }^{2}$ Key Laboratory of Enhanced Oil and Gas Recovery of Ministry of Education, Daqing Petroleum \\ Institute, Daqing, Heilongjiang 163318, China \\ Email: yinhj7176@126.com
}

\begin{abstract}
In general, the shape of practical oil reservoir is irregular and the boundary condition is complex. What is more, there may be one or several areas of different permeabilities in it. The irregular shape, the complex boundary condition and the existence of several areas of different permeabilities inevitably affect the pressure distribution of the reservoir. A considerable amount of time and effort has spent on this issue. So to calculate and analyze the pressure of this kind reservoir is of great significance.

In this paper, the main work have been done as follows:

At first, the mathematical model which are applicable to steady-state flow in arbitrary shaped heterogeneous reservoir is built up and solved by he Boundary Element Method (BEM) with dividing the whole reservoir into several areas based on different permeabilities.

Secondly, the radial flow of two areas in heterogeneous reservoir is chosen to verify the validity of this method. By using this method to solve the steady-state flow in the radial heterogeneous reservoir, the comparison between the numerical solutions and the analytical solutions is done to ascertain the exactness of this method. The pressure distribution curve is also drawn. By analyzing, it is found that this special Boundary Element Method of dividing the whole reservoir into several areas is not only applicable and correct but also quite precise in solving the problems of heterogeneous reservoir for steady-state flow.

At last, the heterogeneous reservoir with several areas, arbitrary shape and complex boundary condition is also be solved by this method. The pressure value at any interior point can be obtained, so the pressure distribution map can also be drawn. By analyzing, it is found that the pressure distribution presents as a ladder because of several areas, which have different permeabilities and deplete energy differently. And the bigger the ratio of permeability is, the more apparent the degree is.
\end{abstract}

\section{REFERENCES}

1. Yin $\mathrm{H} \mathrm{J}, \mathrm{He} Y \mathrm{~F}$. Pressure transient analysis of heterogenous reservoirs with impermeability barrier using perturbation boundary element method. Journal of Hydrodynamics Ser. B, 2005; 17(1)102109, 2002 (in Chinese)

2. Kikani J, Horne R. Pressure Transient analysis of arbitrary shaped reservoirs with the boundary element method. SPE 18159, 1988: 519-529

3. Yang D Q, Zhao Z S. The Theory and Application for Boundary Element Method. Beijing: Beijing Institute of Technology Press, 2002 (in Chinese) 